\title{
Aversiveness without pain: Potentiation of imaginal and auditory effects of blackboard screeches
}

\author{
DAVID J. ELY \\ Porterville State Hospital Porterville, California 93257
}

\begin{abstract}
Sixteen subjects were presented with a mixed sequence of eight tones (T) and eight screeches (S) produced by scratching a blackboard. Half of the subjects were told how the screeches were produced (Cond I), while half were told that some sounds were complex (Cond NI). In Cond I, palmar skin potentials increased to $\mathrm{S}$ and remained the same to $\mathrm{T}$ with repeated presentations. In Cond NI, the difference between $\mathrm{S}$ and $\mathrm{T}$ remained constant over repeated presentations. Only four subjects reported having gooseflesh or unpleasant sensations. The combined effect of the screech and information regarding the way screech was produced appeared to be one of sensitization over repeated presentations. This suggested that anecdotal accounts of the effect of imaginal processes upon the reaction to this sort of sound were correct.
\end{abstract}

In an early report, Lindsley and Sassaman (1938) investigated autonomic responses of an individual who claimed to be able to voluntarily produce gooseflesh (hereafter called horripilation). They concluded that the subject's voluntary control of horripilation was not due to meditational effects of striate muscular activity.

When a complex sound containing irregularly fluctuating high-frequency components reaches certain, as yet unanalyzed, values, human listeners report unpleasant sensations and horripilation. According to anecdotal information, these unpleasant sensations do not occur at the sense organ (the ears) but on various bodily surfaces. In this respect, the stimulus differs from other sorts of unpleasant stimulation. Some informants indicate that unpleasant sensations can be intensified by imagining the production of such sounds by, for example, scratching a blackboard with the fingernails. ${ }^{1}$ It is possible that the response to the sound is based upon a history of conditioning, in which the unpleasant tactual sensation of scratching a blackboard has served as the UCS, and the sound produced has acquired the properties of a CS. However, this speculation is premature.

Although almost all informants attribute the unpleasant sensations to the sound, it has not been demonstrated that such sounds differ from any other sound with respect to autonomic effects on humans. Accordingly, it is necessary to evaluate some of the phenomena described in anecdotal accounts, including those regarding the effects of imaginal processes on responses to such sounds.

In the present study, palmar skin potentials were recorded while subjects listened to tones and blackboard screeches. An instructional manipulation intended to

The views and procedures presented here are the author's and do not necessarily reflect those of Porterville State Hospital or the California Department of Health. facilitate autonomic responses to screech sounds was used. This manipulation was presumed to be analogous to imaginal processes described by informants.

\section{METHOD}

A total of eight female and eight male employees of Porterville State Hospital volunteered to participate in the experiment in their own time. Female and male subjects were equally divided within the instructional conditions.

Stimuli were presented via a Sony Model TC105A tape recorder and binaural headphones. The sound system had a net high frequency loss of $5 \mathrm{~dB}$ at $12 \mathrm{kHz}$. Tones were generated by a Dynascan oscillator at $4 \mathrm{kHz}$. Screeches were generated by drawing a small plastic tab across a blackboard. Both screeches and tones were recorded so that when played at a specified gain, the amplitude of the tone was $70 \mathrm{~dB}$ and of the screech was $65 \mathrm{~dB}$. Duration of both was $5 \mathrm{sec}$, and interstimulus interval was $20-40 \mathrm{sec}$, with a $30-\mathrm{sec}$ mean. Two tapes consisted of eight presentations of the tone (T) and eight of the screech (S) in a recurring $S, T, T, S$ order. Two other tapes consisted of a recurring $T, S, S, T$ order of presentation.

A Beckman-type RS oscillograph monitored palmar skin potentials, using silver electrodes and starch-saline paste. A .3-sec time constant was maintained on the input of the preamplifier.

Subjects were told that the study investigated the relationship between sounds and physiological functioning. All subjects were told that they would hear two types of sound and that the investigation compared complex to simple sound. Subjects assigned to the informed condition (I) were told that some of the sounds were produced by scratching a blackboard. Subjects assigned to the not informed (NI) condition were given no information about the way sounds were produced. Following attachment of headphones and electrodes, subjects were told to remain relaxed, alert, as motionless as possible, and to listen to the sounds. One of the four tapes was then presented.

\section{RESULTS}

Skin potential amplitude was defined as the largest deflection occurring from $.5 \mathrm{sec}$ to $5.0 \mathrm{sec}$ following stimulus onset. Mean skin potentials over four blocks of two presentations are shown in Figure 1. The method of 


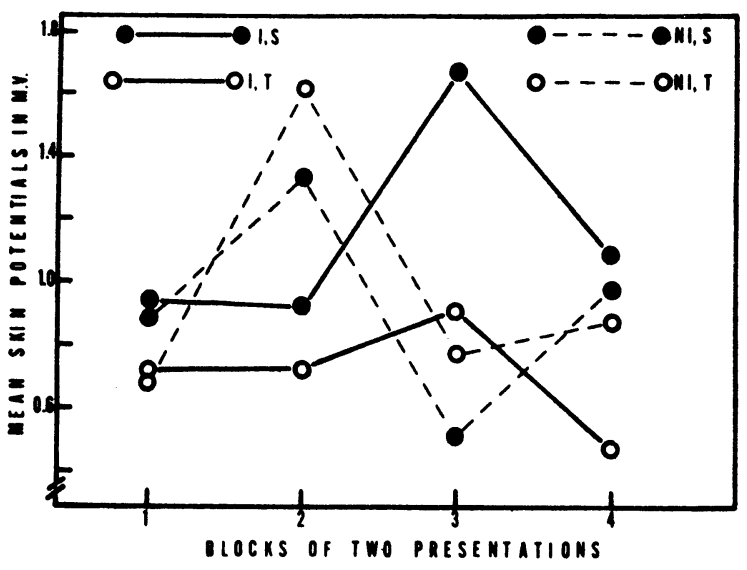

Figure 1. Mean skin potential over blocks of two presentations. $S=$ screech, $T=$ tone,$I=$ informed condition, $\mathrm{NI}=$ not informed condition.

partitioning 16 trials into two sets of eight presentations was such that Presentation 1 of " $S$ " consisted of all cases where "S" was presented for the first time, regardless of whether it was preceded by a "T", and so on.

Subjects in Condition I yielded smaller responses than subjects in Condition NI during the first half of the presentations, while the obverse was true during the second half of the presentations. This was a significant $(\mathrm{p}<.05)$ Instruction by Presentations interaction $[\mathrm{F}(7 / 210)=2.43$, EMS $=1.03]$. The difference between $\mathrm{S}$ and $\mathrm{T}$ increased over presentations in Condition I, but it did not do so in Condition NI. This was a significant Instructions by Stimulus by Presentation interaction $[F(7 / 210)=3.72, \quad E M S=1.03]$. No other effects achieved significance.

Only four subjects reported horripilation or unpleasant sensations during the experiment. All of these occurred in response to screeches. Of the four subjects reporting, three were in Condition I, and one was in Condition NI.

\section{DISCUSSION}

During the course of successive presentations, the difference between responses to screeches and tones became greater in the informed condition but did not shange in the not informed condition. The fact that this effect developed during later presentations rather than occurring during earlier presentations suggested that some sort of sensitization to screech sounds was taking place. This effect seems to disarm the possible criticism that the effect of the instructional manipulation could be accounted for by appealing to the well-established fact that responses to stimuli are influenced by social sets and expectancies. If this critique were correct, the difference between responses to the two types of sound should have appeared during earlier presentations and should not have developed during later presentations.

Despite the high frequency limitations of tape recording equipment, it was possible to demonstrate a difference in autonomic responses to the two types of stimuli, including a limited amount of horripilation in response to some screech sounds. The success of this demonstration was dependent upon the use of a moderate instructional manipulation, which was presumed to influence imaginal processes such as reported in anecdotal accounts.

Subjects in the not informed condition reached a peak of responding on the third and fourth presentations, while subjects in the informed condition reached the same sort of peak on the fifth and sixth presentations. The fact that this occurred with both types of sounds rules out the possibility that the peaks might have been the result of incidental variations in stimulus parameters. It is probable that the use of the mixed screech tone sequence occurring in a predictable order could have produced this effect. The use of the mixed sequence of dissimilar sounds may also account for the lack of apparent habituation observed throughout the experiment.

\section{REFERENCE}

Lindsley, D. B., \& Sassaman, W. H. Autonomic activity and brain potentials associated with "voluntary" control of the pilomotors (MM. arrectores pilorum). Journal of Neurophysiology, 1938, 1, 342-349.

\section{NOTE}

1. The author wishes to apologize for discomfort which may be caused by reading this paper. He would also like to thank Dr. Raymond Weitzman, Department of Linguistics, for the preparation of spectrograms of the screech sound used in the experiment. Analysis of the sound used was beyond the scope of the present paper, but copies of the analysis may be obtained by contacting the author.

(Received for publication June 9, 1975.) 